https://doi.org/10.15407/ujpe63.3.255

A.N. VASILEV

Taras Shevchenko National University of Kyiv,

Department of Theoretical Physics, Physics Faculty

(64/13, Volodymyrska Str., Kyiv 01601, Ukraine; e-mail: vasilev@univ.kiev.ua)

\title{
ANALYTICAL APPROACH FOR CALCULATING THE CHEMOTAXIS SENSITIVITY FUNCTION
}

\begin{abstract}
We consider the chemotaxis problem for a one-dimensional system. To analyze the interaction of bacteria and an attractant, we use a modified Keller-Segel model, which accounts for the attractant absorption. To describe the system, we use the chemotaxis sensitivity function, which characterizes the nonuniformity of the bacteria distribution. In particular, we investigate how the chemotaxis sensitivity function depends on the concentration of an attractant at the boundary of the system. It is known that, in the system without absorption, the chemotaxis sensitivity function has a bell shape maximum. Here, we show that the attractant absorption and special boundary conditions for bacteria can cause the appearance of an additional maximum in the chemotaxis sensitivity function. The value of this maximum is determined by the intensity of absorption.
\end{abstract}

Ke ywords: chemotaxis, attractant, bacteria, absorption.

\section{Introduction}

It is well known that when a bacterium like $E$. coli is placed in some substance (which is called attractant) with the concentration gradient, then the bacterium moves toward the attractant gradient. This phenomenon is known as the chemotaxis [1-6]. Although many interesting and significant results have been obtained in this area (e.g., see [7-22]), we are going to pay some attention to the process of the bacteria redistribution in the presence of an attractant.

Frequently, we do not need to know the exact spatial distribution of bacteria in the system. What we need is just some numerical characteristics that could be measured in an experiment. One of them is the chemotaxis sensitivity function [6]. Namely, we will focus our attention on the one-dimensional system with an attractant that is injected into the system at the left boundary. Technically, it could be done by placing a capillary with the attractant [6]. The system also contains bacteria which can interact with the attractant. To investigate the system, we will use the methodological approach that was developed in [6]. In particular, our main goal will be the chemotaxis sensi-

(C) A.N. VASILEV, 2018

ISSN 2071-0194. Ukr. J. Phys. 2018. Vol. 63, No. 3 tivity function, which characterizes the nonuniformity of the bacteria distribution. As was shown in [6], this function depends on the attractant concentration in a nontrivial way. Those results were obtained for the system with the linear distribution of an attractant, that can be realized in the case where bacteria do not absorb the attractant. Here, we consider a more complex situation. But first of all, we will make some comments about how we are to define the chemotaxis sensitivity function.

Consider a one-dimensional system, whose spatial coordinate $x$ can change from 0 to $L$ (i.e. $0 \leq x \leq$ $\leq L)$. Let it be that a function $b(x)$ determines the spatial distribution of bacteria. We also assume that the system contains an attractant, and it is injected into the system at the left boundary with the help of some special capillary. The capillary, as is supposed, is placed within the region $0 \leq x \leq r_{c}$, where $r_{c}$ is the size of the capillary. At the right boundary of the system, the concentration of the attractant is fixed at a level lower than it is at the left boundary. Then the concentration of bacteria should be the highest at the left boundary, within the region of the capillary.

For the above-described one-dimensional system, the chemotaxis sensitivity function can be defined as 
follows [6]:

$F=\frac{L P_{b}}{r_{c}}-1$

The parameter $P_{b}$ in formula (1) is the probability to find a bacterium within the region $0 \leq x \leq r_{c}$, and it is determined like this:

$P_{b}=\frac{\int_{0}^{r_{c}} b(x) d x}{\int_{0}^{L} b(x) d x}$.

Actually, in formula (1), we have the ratio of the probability $P_{b}$ for a bacterium to be within the region of the capillary, to the probability $\frac{r_{c}}{L}$ for a bacterium to be within the region of the capillary, if bacteria are distributed uniformly in the system. Thus, the chemotaxis sensitivity function is a numerical characteristics giving some notion of the bacteria distribution. If bacteria are distributed uniformly, then $F=0$. In the general case, it can be of any sign. The greater the chemotaxis sensitivity function (by the modulus), the more nonuniform is the bacteria distribution.

It is also notable that, in the limiting case where $r_{c} \ll L$, we can rewrite the expression for the chemotaxis sensitivity function as [6]

$F=\frac{L b(0)}{\int_{0}^{L} b(x) d x}-1$.

Thus, to calculate the chemotaxis sensitivity function, it is enough to know the total amount of bacteria in the system and the concentration of bacteria at the left boundary.

Next, we consider a model that describes the onedimensional system with bacteria and an attractant. It is assumed that bacteria are redistributed according to the attractant gradient, and the attractant is absorbed by bacteria. As was mentioned above, to characterize such a system, we will use the chemotaxis sensitivity function.

\section{Basic Model}

To calculate the chemotaxis sensitivity function for the system with bacteria that absorb the attractant, we use a model of the Keller-Segel kind [23-25]. As is known, the classical Keller-Segel model is based on the nonlinear partial differential equations [23]

$\partial_{t} a(t, \mathbf{r})=D_{a} \Delta a(t, \mathbf{r})+f_{1}(a, b)$,

$\partial_{t} b(t, \mathbf{r})=D_{b} \Delta b(t, \mathbf{r})+f_{2}(a, b)$,

where $\partial_{t}$ denotes the partial derivative with respect to time $t, b(t, \mathbf{r})$ stands for the bacteria concentration, and $a(t, \mathbf{r})$ is the attractant concentration. The parameters $D_{a}$ and $D_{b}$ are the diffusion coefficients. The function $f_{1}(a, b)$ accounts for the absorption and the secretion of the attractant, and the function $f_{2}(a, b)$ defines the chemotactic flow of bacteria. If these functions are specified (as well as the boundary and initial conditions), then we can solve the system of equations (4)-(5), at least in a numerical form [26-34]. As was mentioned above, the function $f_{1}(a, b)$ describes the attractant absorption (the attractant secretion will be accounted by the boundary conditions). Our assumptions concerning this function are as follows:

- the intensity of the attractant absorption is proportional to the bacteria density;

- at low attractant concentration, the intensity of the attractant absorption is proportional to the attractant concentration;

- at high attractant concentrations, the intensity of the attractant absorption does not depend on the attractant concentration.

All these allow us to consider the function $f_{1}(a, b)$ to be like this:

$f_{1}(a, b)=-k_{1} \frac{a b}{a_{1}+a}$,

where $k_{1}$ and $a_{1}$ are phenomenological parameters of the model. Our basic assumption for the function $f_{2}(a, b)$ is that the bacteria flux $j_{b}$ is determined by the bacteria concentration, its gradient, and the gradient of the attractant. In particular, we use the following formula for the bacteria flux:

$j_{b}=-D_{b} \nabla b+b \varphi(a) \nabla a$.

The first term in Eq. (7) determines the flow of bacteria due to the diffusion, and, thus, $D_{b}$ stands for the diffusion coefficient. The second term determines the bacteria flow caused by the inhomogeneity of the attractant distribution. It is supposed that this particular term is proportional to the bacteria concentration and to the attractant gradient. This term de-

ISSN 2071-0194. Ukr. J. Phys. 2018. Vol. 63, No. 3 
pends also on the attractant concentration in a nonlinear way. To account this dependence, we use the function $\varphi(a)$.

Thus, we can rewrite the equation that determines the temporal evolution of the bacteria distribution. In particular, we have the following:

$\partial_{t} b=D_{b} \Delta b-\nabla(b \varphi(a) \nabla a)$.

In the stationary case, we get the equation, which ties the bacteria distribution and the attractant distribution:

$D_{b} \Delta b-\nabla(b \varphi(a) \nabla a)=0$.

It can be reduced to the first-order differential equation of the form

$D_{b} \nabla b-b \varphi(a) \nabla a=0$.

For the one-dimensional geometry (where $0 \leq x \leq L$ ), this equation with the boundary condition

$\left.j_{b}\right|_{z=0}=0$

(which means the zero bacteria flux at the left boundary) produces the next formula determining the relation between the bacteria concentration $b(x)$ and the attractant concentration $a(x)$ :

$b(x)=A \exp \left(\frac{1}{D_{b}} \int \varphi(a) d a\right)$.

The constant of integration $A$ should be determined by another boundary condition for the bacteria distribution, which we will consider and discuss later.

To make some quantitative analysis, we have to specify the function $\varphi(a)$ (and by it, the function $\left.f_{2}(a, b)\right)$. Here, we take into account that the chemotaxis bacteria flow is proportional to the attractant gradient at low attractant concentrations, it is decreased (down to zero) with increasing the attractant concentration, and it is also proportional to the bacteria concentration. According to this, we can present the function $f_{2}(a, b)$ in the form

$f_{2}(a, b)=k_{2} \nabla\left(\frac{b \nabla a}{\left(a_{2}+a\right)^{2}}\right)$

with phenomenological parameters $k_{2}$ and $a_{2}$. Thus, the function $\varphi(a)$ is as follows:

$\varphi(a)=\frac{k_{2}}{\left(a_{2}+a\right)^{2}}$.

ISSN 2071-0194. Ukr. J. Phys. 2018. Vol. 63, No. 3

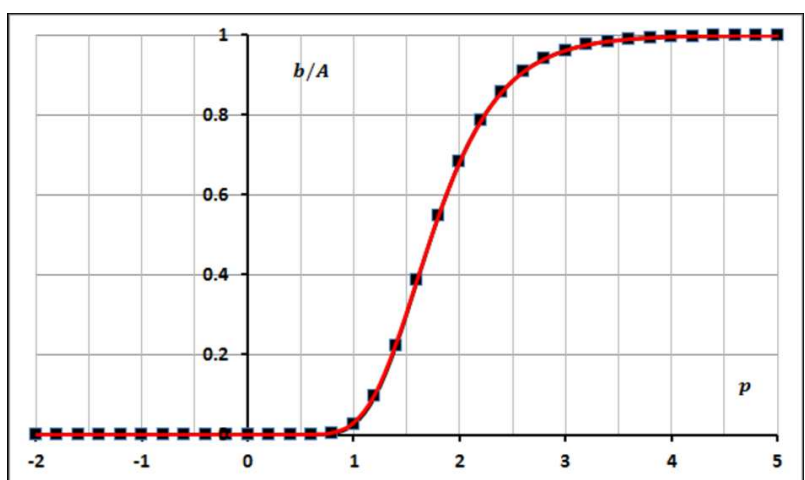

Fig. 1. Bacteria concentration as a function of the attractant concentration. It is taken that $a=a_{2} \times 10^{p}$. The solid line shows the dependence by formula (15). The points (squares) correspond to the dependence that is presented with formula (16). It is also taken that $N \approx 38.56$ and $c_{2} / c_{1} \approx 166.67$

Then relation (12) between the bacteria and attractant concentrations can be rewritten like this:

$b(x)=A \exp \left(-\frac{k_{2}}{D_{b}} \frac{1}{a_{2}+a}\right)$.

Equation (15) gives the relation between the bacteria and attractant concentrations. We can compare it to the similar relation that was obtained in [6] in the particular case with a linear distribution of the attractant in the system (the system without absorption). It is of the form [6]

$b(x)=A\left(\frac{c_{1}+a(x)}{c_{2}+a(x)}\right)^{N}$,

where $A$ is a normalization constant (just the same as in Eq. (15)), $c_{1,2}$ and $N$ are parameters of the model used in [6]. Formally, relations (15) and (16) are different. Nevertheless, numerical estimations for the dimensionless parameter $N$ give that $N \gg 1$ for the real systems. So, if we perform the limiting transition $N \rightarrow \infty$, then formula (16) yields:

$b(x) \approx A \exp \left(-N \frac{c_{2}-c_{1}}{c_{2}+a(x)}\right)$.

We see that if $c_{2}=a_{2}$ and $k_{2}=D_{b} N\left(c_{2}-c_{1}\right)$, then Eqs. (15) and (17) determine the same dependences. To estimate and to compare the dependences that are given by Eqs. (15) and (16), we use the following values for the parameters (according to the data in [6]): $N \approx 38.56$ and $c_{2} / c_{1} \approx 166.67$. Figure 1 presents the bacteria concentration as a function of 
the attractant concentration, which is calculated according to formulae (15) and (16). For the attractant concentration, it is taken that $a=a_{2} \times 10^{p}$. As we can easily see from Fig. 1, formulae (15) and (16) give actually the same dependences.

\section{Attractant Distribution}

In view of the previous results, we get the following differential equation for the stationary distribution of the attractant:

$D_{a} \Delta a(x)-k_{1} A \exp \left(-\frac{k_{2}}{D_{b}} \frac{1}{a_{2}+a}\right) \frac{a(x)}{a_{1}+a(x)}=0$.

It should be supplemented with some boundary conditions for the attractant concentration, and with an additional condition for the bacteria distribution function. We consider the boundary conditions for the attractant distribution, when the attractant concentration is fixed at the boundaries. This means that

$a(x=0)=C_{0}$,

$a(x=L)=C_{1}$,

and the parameters $C_{0}$ and $C_{1}$ (we assume that $C_{0} \geq$ $\geq C_{1}$ ) are given.

Our next step deals with redefining some parameters. In particular, for the sake of simplicity, we use the substitutions $x=L z$ and $a(x)=a_{2} s(z)$. Then we get the following equation:

$s^{\prime \prime}(z)-\alpha A \exp \left(-\frac{\beta}{1+s(z)}\right) \frac{s(z)}{\lambda+s(z)}=0$,

where we have used the parameters $\alpha=\frac{k_{1} L^{2}}{D_{a} a_{2}}, \beta=$ $=\frac{k_{2}}{D_{b} a_{2}}$ and $\lambda=\frac{a_{1}}{a_{2}}$. The boundary conditions are transformed to these ones:

$$
\begin{aligned}
& s(z=0)=\frac{C_{0}}{a_{2}} \equiv \gamma_{0}, \\
& s(z=1)=\frac{C_{1}}{a_{2}} \equiv \gamma_{1} .
\end{aligned}
$$

In this case, the chemotaxis sensitivity function $F$ is determined by the relation

$$
F=\frac{b(0)}{\int_{0}^{1} b(z) d z}-1,
$$

where the bacteria distribution is given by the expression

$b(z)=A \exp \left(-\frac{\beta}{1+s(z)}\right)$

Thus, to solve the problem and to find the value of the chemotaxis sensitivity function (basing on some additional restriction imposed on the bacteria distribution function $b(z)$ ), we have to specify the constant $A$ in relation (25), to solve then Eq. (21) with the boundary conditions (22) and (23), and, after that, to calculate the chemotaxis sensitivity function $F$ according to relation (24).

\section{Chemotaxis Sensitivity Function}

Next, we consider the chemotaxis sensitivity function and, in particular, clarify how it depends on the attractant concentration at the left boundary of the system. It is understood that the chemotaxis sensitivity function reads

$F=\frac{b(0)}{\int_{0}^{1} b(z) d z}-1=\frac{\exp \left(-\frac{\beta}{1+\gamma_{0}}\right)}{\int_{0}^{1} \exp \left(-\frac{\beta}{1+s(z)}\right) d z}-1$,

and it is formally independent of $A$. Nevertheless, the solution for the bacteria distribution $s(z)$ is determined by Eq. (21), which contains the parameter $A$. So, the chemotaxis sensitivity function depends implicitly on how we determine $A$. It depends, in turn, on the restriction we apply for the concentration $b(z)$ of bacteria. Here, we will consider three regimes that specify the distribution of bacteria:

- the concentration of bacteria at the right boundary is fixed;

- the total amount (or mass) of bacteria in the system is fixed;

- the concentration of bacteria at the right boundary is changed with changing the attractant concentration, to supply the parameter $A$ to be fixed.

Fixing the bacteria concentration at the right boundary, $b(1)=B_{1}$, gives the restriction

$A \exp \left(-\frac{\beta}{1+\gamma_{1}}\right)=B_{1}$

Let the value of the parameter $B_{1}$ be given. Then, to solve the problem, we have to solve Eq. (21), which

ISSN 2071-0194. Ukr. J. Phys. 2018. Vol. 63, No. 3 
is transformed, in this case, to the following:

$s^{\prime \prime}(z)-\alpha B_{1} \exp \left(\frac{\beta}{1+\gamma_{1}}\right) \exp \left(-\frac{\beta}{1+s(z)}\right) \frac{s(z)}{\lambda+s(z)}=0$.

Then, knowing the distribution $s(z)$, we calculate the chemotaxis sensitivity function according to Eq. (24). Figure 2 illustrates how the chemotaxis sensitivity function depends on the attractant concentration at the left boundary of the system. In particular, we take $\gamma_{0}=10^{p}$ and $\gamma_{1}=\xi \gamma_{0}$, where $\xi=0.75$ is fixed, and the parameter $p$ changes from -3 to 3 . We also took $\lambda=10$ and $\beta=38.56$.

As we can see, the dependence of the chemotaxis sensitivity function on the attractant concentration at the left boundary (more precisely, on the parameter $p$ ) has a bell shape maximum. The value of the maximum depends on the parameters of the model. But the matter of fact is that the maximum exists, and that it is the only maximum.

Fixing the total amount of bacteria in the system $\int_{0}^{1} b(z)=B_{2}$ gives the following restriction:

$A \int_{0}^{1} \exp \left(-\frac{\beta}{1+s(z)}\right) d z=B_{2}$.

In this case, to find the chemotaxis sensitivity function, we have actually to solve a system of equations. The first one is Eq. (21). It contains the parameter $A$. On the other hand, this parameter is to satisfy relation (29), which contains, in turn, the solution $s(z)$ of Eq. (21). Numerical calculations for this problem show that the dependence of the chemotaxis sensitivity function on the attractant concentration (at the left boundary) is the same qualitatively as in the previous case (when we fix the bacteria concentration at the right boundary). Figure 3 compares these two cases. It contains the plots for the chemotaxis sensitivity functions that were calculated a) with a fixed bacteria concentration at the right boundary, and b) with a fixed total amount of bacteria in the system.

The third scenario is when we change the concentration of bacteria at the right boundary synchronously with changing the attractant concentration at the left boundary. In particular, we take the

ISSN 2071-0194. Ukr. J. Phys. 2018. Vol. 63, No. 3

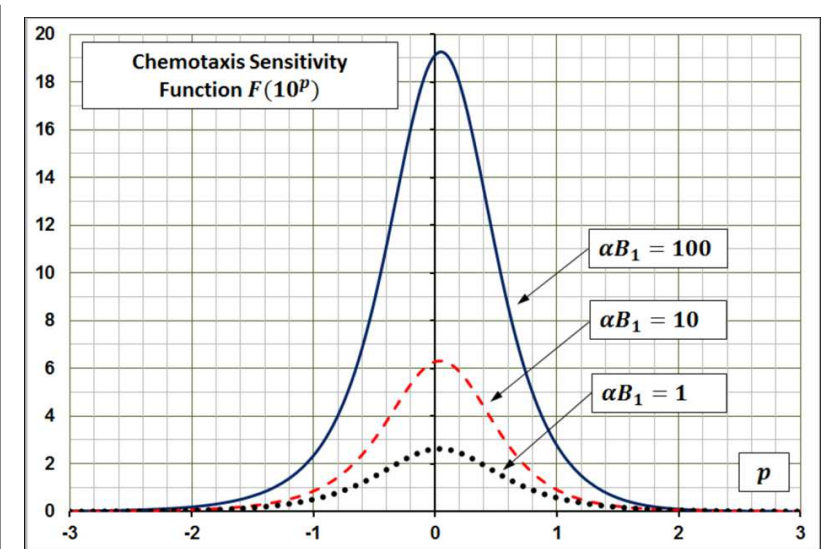

Fig. 2. Dependence of the chemotaxis sensitivity function on the attractant concentration at the left boundary. It is taken that $\gamma_{0}=10^{p}, \gamma_{1}=\xi \gamma_{0}, \lambda=10$, and $\beta=38.56$. The dotted line is for the value $\alpha B_{1}=1$, the dashed line is for the value $\alpha B_{1}=10$, and the solid line is for the value $\alpha B_{1}=100$

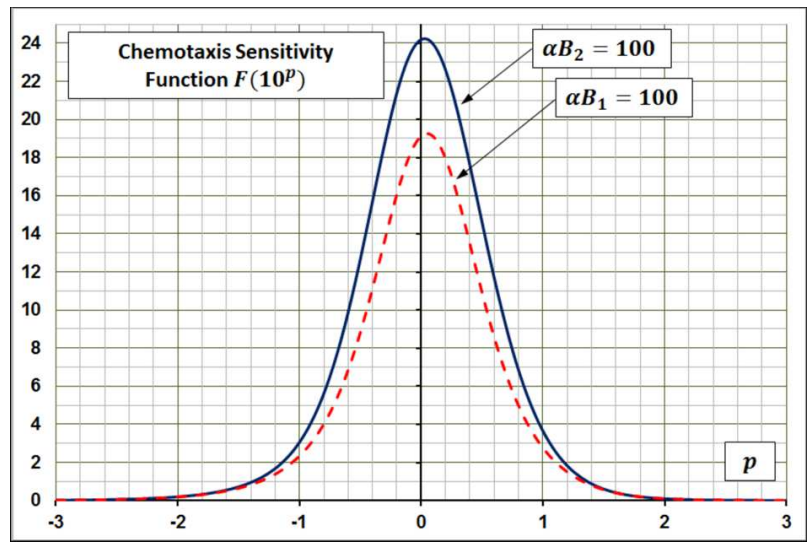

Fig. 3. Dependence of the chemotaxis sensitivity function on the attractant concentration at the left boundary. It is taken that $\gamma_{0}=10^{p}, \gamma_{1}=\xi \gamma_{0}, \lambda=10$, and $\beta=38.56$. The dashed line is for the value $\alpha B_{1}=10$ (the bacteria concentration at the right boundary is fixed), and the solid line is for the value $\alpha B_{2}=100$ (the total amount of bacteria is fixed)

following boundary condition for the bacteria concentration:

$b(1)=B_{3} \exp \left(-\frac{\beta}{1+\gamma_{1}}\right)$

This gives the condition $A=B_{3}$ for solving Eq. (21). Figure 4 shows how the chemotaxis sensitivity function looks like in this case. The most important thing is that it may have two maxima. In particular, increasing the value of the product $\alpha B_{3}$ leads to the 


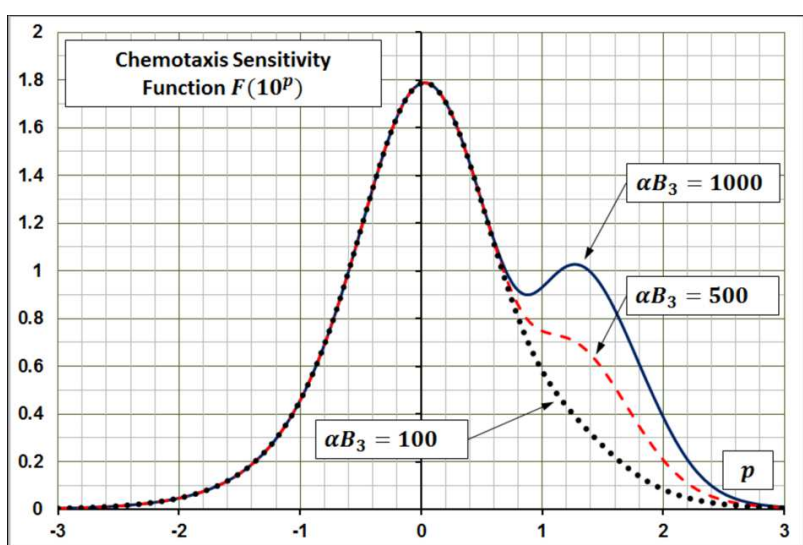

Fig. 4. Dependence of the chemotaxis sensitivity function on the attractant concentration at the left boundary. It is taken that $\gamma_{0}=10^{p}, \gamma_{1}=\xi \gamma_{0}, \lambda=10$, and $\beta=38.56$. The dotted line is for the value $\alpha B_{3}=100$, the dashed line is for the value $\alpha B_{3}=500$, and the solid line is for the value $\alpha B_{3}=1000$

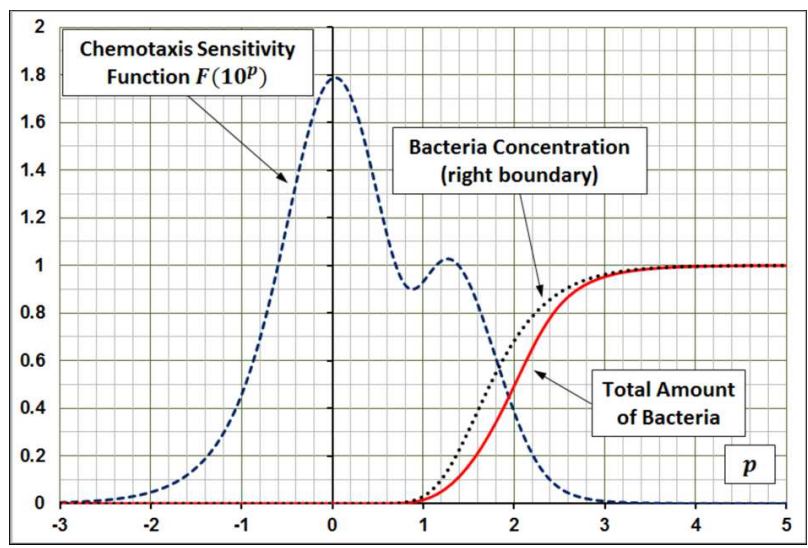

Fig. 5. Chemotaxis sensitivity function and the bacteria total amount. It is taken that $\gamma_{0}=10^{p}, \gamma_{1}=\xi \gamma_{0}, \lambda=10$, $\beta=38.56$, the value $\alpha B_{3}=1000$. The dotted line shows the concentration of bacteria at the right boundary, the solid line shows the total amount of bacteria in the system, and the dashed line demonstrates how the chemotaxis sensitivity function depends on the attractant concentration at the left boundary of the system. The bacteria concentration is taken without multiplier $B_{3}$ (which is a constant)

appearance of an additional maximum at high concentrations of the attractant. So, it is clear that this effect is caused by the attractant absorption. It is also notable that the way we take the boundary condition for bacteria is of importance. Figure 5 illustrates how the total amount of bacteria and the bacteria concentration at the right boundary are changed with chang-

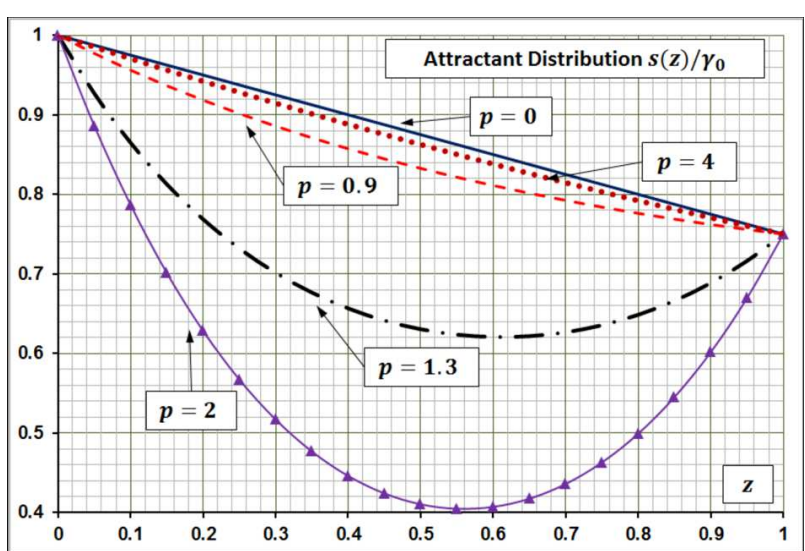

Fig. 6. Attractant distribution $s(z) / \gamma_{0}$ for the different values of the parameter $p$ (it is taken that $\gamma_{0}=10^{p}, \gamma_{1}=\xi \gamma_{0}, \lambda=10$, $\beta=38.56$, and the value $\left.\alpha B_{3}=1000\right)$ : the solid line is for the value $p=0$, the dashed line is for the value $p=0.9$, the dashdotted line is for the value $p=1.3$, the solid line with triangular markers is for the value $p=2$, and the dotted line is for the value $p=4$

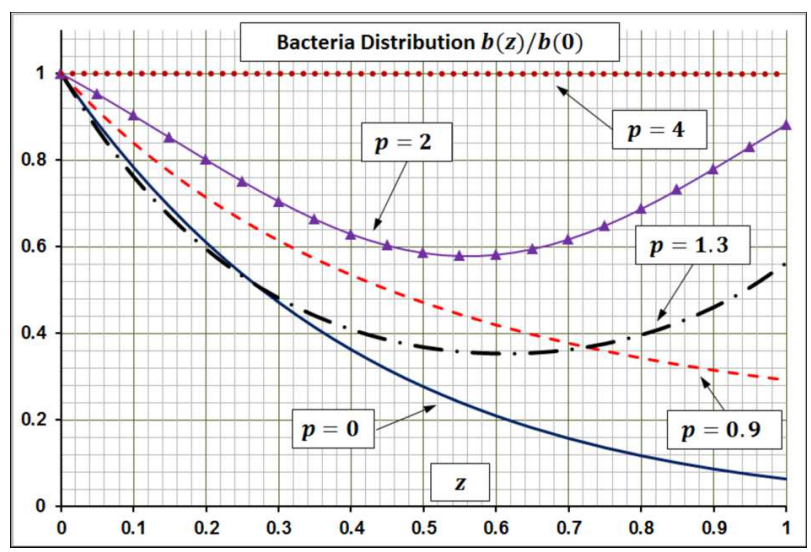

Fig. 7. Bacteria distribution $b(z) / b(0)$ for the different values of the parameter $p$ (it is taken that $\gamma_{0}=10^{p}, \gamma_{1}=\xi \gamma_{0}$, $\lambda=10, \beta=38.56$, the value $\left.\alpha B_{3}=1000\right)$ : the solid line is for the value $p=0$, the dashed line is for the value $p=0.9$, the dash-dotted line is for the value $p=1.3$, the solid line with triangular markers is for the value $p=2$, and the dotted line is for the value $p=4$

ing the attractant concentration at the left boundary of the system. All these characteristics are normalized to the $B_{3}$ constant. For the sake of simplicity, Fig. 5 also contains the plot for the chemotaxis sensitivity function. What we can see is that the region of the second additional maximum coincides with the region, where the bacteria concentration is increased. 


\section{Discussion}

So, when we fix the bacteria concentration at the right boundary or the total amount of bacteria in the system, then the chemotaxis sensitivity function has a bell shape maximum. It has a quite obvious explanation [6]. Matter of fact is that when we increase the attractant concentration, then the gradient of the attractant concentration is increased as well. Due to increasing the gradient, the bacteria distribution becomes more nonuniform, and, thus, the chemotaxis sensitivity function is increased. But, at high levels of the attractant concentration, the bacteria reaction on the attractant gradient is decreased. In other words, bacteria "do not feel" the gradient, when the attractant concentration is significant. Thus, the bacteria distribution becomes more uniform, and the chemotaxis sensitivity function is decreased. From physiological point of view, this can be explained in the way a bacterium behaves in the system with an attractant. What we know is that every bacterium has receptors which can interact with an attractant (e.g., see [6] and references therein). The amount of receptors that are in interaction with the attractant determines the methylation level of the bacterium [6]. Any bacterium moves straight with a constant velocity. But, from time to time, it changes the direction of its motion. These acts are called tumbles. It is generally accepted that the new direction of motion is selected randomly. The frequency of tumbles depends on the methylation level of the bacterium. The greater the methylation level, the smaller the tumble frequency. Actually, this is the simplified mechanism of how bacteria behave within the system with an attractant. It is clear that if the attractant concentration is high enough, then the methylation level can be at the highest possible level. Thus, bacteria cannot react to the changes of the attractant concentration [6].

In our model, the effect of the bell shape chemotaxis sensitivity function can be explained, if we account for the relation between the bacteria and attractant concentrations (see equation (15) and Fig. 1). It gives that when the attractant concentration is high, then the bacteria concentration is at the saturation level. The further increase of the attractant concentration does not change the bacteria concentration. Thus, the presence of the gradient of the attractant concentration is not tested by bacteria.
The situation with two maxima of the chemotaxis sensitivity function is explained in the way that when we change the bacteria concentration at the right boundary, then we actually change the total amount of bacteria in the system. If bacteria did not absorb an attractant, then the change of their total amount would not affect the attractant distribution. In turn, it would not change the bacteria distribution. Due to the attractant absorption, increasing the total amount of bacteria in the system changes the attractant distribution. The situation is illustrated in Fig. 6, where the plots are presented for the attractant distribution $s(z) / \gamma_{0}$ for different values of the parameter $p$. In particular, we can see that, at the value $p=0$, the distribution is almost linear. With the further increase of the parameter $p$, the distribution becomes more nonlinear, but then it comes back to the almost linear trend. Say, for the value $p=4$ (the solid line in Fig. 6), the attractant distribution is very close to the distribution for the value $p=0$ (the dotted line in Fig. 6).

The bacteria distribution is changed in a slightly different way. Figure 7 contains plots for the bacteria distribution $b(z) / b(0)$ in the system for some values of the parameter $p$. For example, for $p=0$ (the solid line in Fig. 7), it decreases monotonously from the left boundary to the right boundary. With increasing the value of the parameter $p$, the slope of the curve is decreased (in Fig. 7, see the dashed line for $p=0.9$ ) simultaneously with appearing the minimum in the distribution (in Fig. 7, see the dash-dotted line for $p=1.3$ and the solid line with triangular markers for $p=2$ ). Then decreasing the value of the minimum gives the almost homogeneous distribution of bacteria in the system (in Fig. 7 , see the dotted line for $p=4)$.

1. J.D. Murray. Mathematical Biology: I. An Introduction (Springer, 2007).

2. H.C. Berg. E. coli in Motion (Springer, 2004).

3. J. Adler. Chemotaxis in bacteria. Science 153, 708 (1966).

4. R.M. Macnab, D.E. Koshland. The gradient-sensing mechanism in bacterial chemotaxis. Proc. Natl. Acad. Sci. USA 69, 2509 (1972).

5. H.C. Berg, D.A. Brown. Chemotaxis in Escherichia coli analysed by three-dimensional tracking. Nature 239, 500 (1972).

6. T. Namba, M. Nishikawa, T. Shibata. The relation of signal transduction to the sensitivity and dynamic range of bacterial chemotaxis. Biophys. J. 103, 1390 (2012). 
7. B.A. Camley, J. Zimmermann, H. Levine, W.-J. Rappel. Emergent collective chemotaxis without single-cell gradient sensing. Phys. Rev. Lett. 116, 098101 (2016).

8. A. Geiseler, P. Hänggi, F. Marchesoni, C. Mulhern, S. Savel'ev. Chemotaxis of artificial microswimmers in active density waves. Phys. Rev. E 94, 012613 (2016).

9. S. Dev, S. Chatterjee. Optimal search in E. coli chemotaxis. Phys. Rev. E 91, 042714 (2015).

10. P. Romanczuk, G. Salbreux. Optimal chemotaxis in intermittent migration of animal cells. Phys. Rev. E 91, 042720 (2015).

11. M. Ebrahimian, M. Yekehzare, M.R. Ejtehadi. LowReynolds-number predator. Phys. Rev. E 92, 063035 (2015).

12. M. Leoni, P. Sens. Polarization of cells and soft objects driven by mechanical interactions: Consequences for migration and chemotaxis. Phys. Rev. E 91, 022720 (2015).

13. M. Meyer, L. Schimansky-Geier. Active Brownian agents with concentration-dependent chemotactic sensitivity. Phys. Rev. E 89, 022711 (2014).

14. J. Zhuang, G. Wei, R.W. Carlsen, M.R. Edwards, R. Marculescu, P. Bogdan, M. Sitti. Analytical modeling and experimental characterization of chemotaxis in Serratia marcescens. Phys. Rev. E 89, 052704 (2014).

15. T. Sagawa, Y. Kikuchi, Y. Inoue, H. Takahashi, T. Muraoka, K. Kinbara, A. Ishijima, H. Fukuoka. Single-cell E. coli response to an instantaneously applied chemotactic signal. Biophys. J. 10, 730 (2014).

16. Y. Tu, T.S. Shimizu, H.C. Berg. Modeling the chemotactic response of Escherichia coli to time-varying stimuli. Proc. Natl. Acad. Sci. USA 105, 14855 (2008).

17. D.A. Clark, L.C. Grant. The bacterial chemotactic response rejects a compromise between transient and steadystate behavior. Proc. Natl. Acad. Sci. USA 102, 9150 (2005).

18. P.G. de Gennes. Chemotaxis: The role of internal delays. Eur. Biophys. J. 33, 691 (2004).

19. R. Tyson, S.R. Lubkin, J.D. Murray. A minimal mechanism of bacterial pattern formation. Proc. Roy. Soc. Lond. B 266, 299 (1999).

20. E.O. Budrene, H. Berg. Complex patterns formed by motile cells of Escherichia coli. Nature 376, 49 (1995).

21. E. Ben-Jacob, O. Schochet, A. Tenenbaum, I. Cohen, A. Czirok, T. Vicsek. Generic modelling of cooperative growth patterns in bacterial colonies. Nature 368, 46 (1994).

22. M.J. Schnitzer. Theory of continuum random walks and application to chemotaxis. Phys. Rev. E 48, 2553 (1993).

23. E.F. Keller, L.A. Segel. Travelling bands of chemotactic bacteria: A theoretical analysis. J. Theor. Biol. 30, 235 (1971)
24. E. Keller, L. Segel. Model for chemotaxis. J. Theor. Biol. 30, 225 (1971).

25. E. Keller, L. Segel. Initiation of slime mold aggregation viewed as an instability. J. Theor. Biol. 26, 399 (1970).

26. M.J. Tindall, S.K. Porter, P.K. Maini, G. Gaglia, J.P. Armitage. Overview of mathematical approaches used to model bacterial chemotaxis. II: Bacterial populations. Bull. Math. Biol. 70, 1570 (2008).

27. F.J. Peaudecerf, R.E. Goldstein. Feeding ducks, bacterial chemotaxis, and the Gini index. Phys. Rev. E 92, 022701 (2015).

28. M. Hilpert. Lattice-Boltzmann model for bacterial chemotaxis. J. Math. Biol. 51, 302 (2005).

29. C. Chiu, F. Hoppensteadt. Mathematical models and simulations of bacterial growth and chemotaxis in a diffusion gradient chamber. J. Math. Biol. 42, 120 (2001).

30. K. Chen, R. Ford, P. Cummings. Mathematical models for motile bacterial transport in cylindrical tubes. J. Theor. Biol. 195, 481 (1998).

31. M. Widman, D. Emerson, C. Chiu, R. Worden. Modelling microbial chemotaxis in a diffusion gradient chamber. Biotech. Bioeng. 55, 191 (1997).

32. R. Lapidus, R. Schiller. Model for the chemotactic response of a bacterial population. Biophys. J. 16, 779 (1976).

33. R. Lapidus, R. Schiller. Bacterial chemotaxis in a fixed attractant gradient. J. Theor. Biol. 53, 215 (1975).

34. R. Lapidus, R. Schiller. A mathematical model for bacterial chemotaxis. Biophys. J. 14, 825 (1974).

Received 12.01.18

\section{O.M. Василъєе}

\section{АНАЛІТИЧНИЙ ПІДХІД}

ЩОДО РОЗРАХУНКУ ФУНКЦІї

чУТЛИВОСТІ ХЕМОТАКСИСУ

$\mathrm{P}$ е $з$ ю м е

Досліджується проблема хемотаксису для одновимірної системи. Аналіз взаємодії бактерій з атрактантом виконується на основі модифікованої моделі Келлера-Зегеля. Для опису системи використовується функція чутливості хемотаксису, яка є характеристикою неоднорідності розподілу бактерій. Зокрема, вивчається питання про те, як функція чутливості хемотаксису залежить від концентрації атрактанту на границях системи. Відомо, що системи без абсорбції атрактанту описуються функцією чутливості хемотаксису з максимумом куполоподібної форми. В даній роботі показано, що абсорбція атрактанту та спеціальні граничні умови можуть зумовити виникнення додаткового максимуму у функції чутливості хемотаксису. Величина такого максимуму визначається інтенсивністю абсорбції атрактанту. 